\title{
Identification of a Novel Splice Variant (c.423-8A>G) of $A P C$ by RNA Sequencing
}

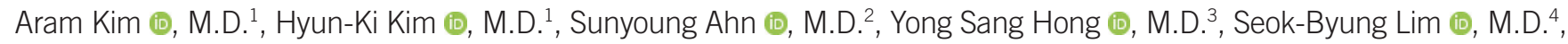

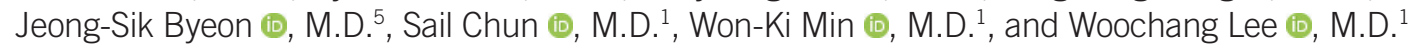 \\ ${ }^{1}$ Department of Laboratory Medicine, University of Ulsan College of Medicine and Asan Medical Center, Seoul, Korea; ${ }^{2}$ Department of Laboratory Medicine, \\ Kangwon National University Hospital, Chuncheon, Korea; Departments of ${ }^{3}$ Oncology, ${ }^{4}$ Surgery, and ${ }^{5}$ Gastroenterology, University of Ulsan College of \\ Medicine and Asan Medical Center, Seoul, Korea
}

\section{Dear Editor,}

Familial adenomatous polyposis (FAP) is an autosomal dominant disorder characterized by the presence of hundreds to thousands of adenomatous polyps throughout the colon and rectum that evolve into colorectal cancer when left untreated [1]. Variants in the adenomatous polyposis coli (APC) gene, a well-known tumor-suppressor gene located on chromosome $5 \mathrm{q} 21$, are responsible for the majority of FAP cases. To date, more than 1,600 pathogenic APC variants have been reported [2]. We identified a novel splice variant of APC in a patient with FAP by next-generation sequencing (NGS) and confirmed its impact on splicing by RNA sequencing. The genetic testing for this patient was performed as a clinical laboratory testing, and RNA sequencing was performed to elucidate the significance of the detected variant. As it is not a part of research subjects, approval from the Institutional Review Board was exempt. Informed consent was obtained from the patient before genetic testing.

In October 2018, a 36 year-old man presented to the Department of Gastroenterology, Asan Medical Center, Seoul, Korea, for further evaluation and management of FAP with adenoma of the ampulla of Vater. His mother, maternal aunt, and maternal cousin had undergone colectomy for FAP in her 50s, her 60s, and his 30s, respectively. Colonoscopy revealed more than 100 small (mostly $<3 \mathrm{~mm}$ ) adenomatous polyps in the colon and rectum, and polypectomy was performed for several large polyps ( $\geq 4 \mathrm{~mm}$ ). Esophagogastroduodenoscopy revealed numerous polyps on the stomach and a mass close to the ampulla of Vater. Biopsy of the mass identified villotubular adenoma with focal high-grade dysplasia, and endoscopic submucosal dissection was performed.

To identify genetic variants causing FAP, we extracted DNA from peripheral blood leukocytes using QIAamp DSP DNA Blood Mini kit (Qiagen, Hilden, Germany) and prepared the library for NGS with a Customized Target Enrichment Kit (Celemics, Seoul, Korea). We conducted NGS with a multigene panel of 128 hereditary tumor-related genes, including colorectal cancer-related genes such as APC, KRAS, MLH1, MSH2, MSH6, MUTYH, PMS2, SMAD4, STK11, and TP53, using a MiSeqDx V2 sequencing kit (Illumina, San Diego, CA, USA) on a Miseq Dx instrument (Illumina). The mean coverage depth was 463.8x, and 204 variants were identified. Except one variant in the intron of $A P C$, all variants were predicted to be benign or likely benign. The identified suspected variant was NC_000005.9 (NM_000038.5):c.423$8 \mathrm{~A}>\mathrm{G}$, which was heterozygous and found in 105 out of 185 reads (56.76\%) (Fig. 1). This variant has never been reported previously. In-silico splice-site prediction algorithms used were
Received: July 12, 2019

Revision received: October 2, 2019

Accepted: January 14, 2020

Corresponding author: Woochang Lee, M.D.

Department of Laboratory Medicine, University of Ulsan College of Medicine and Asan Medical Center, 88 Olympic-ro 43-gil, Songpa-gu, Seoul 05505,

Korea

Tel: +82-2-3010-4506, Fax: +82-2-478-0884

E-mail: wlee1@amc.seoul.kr 


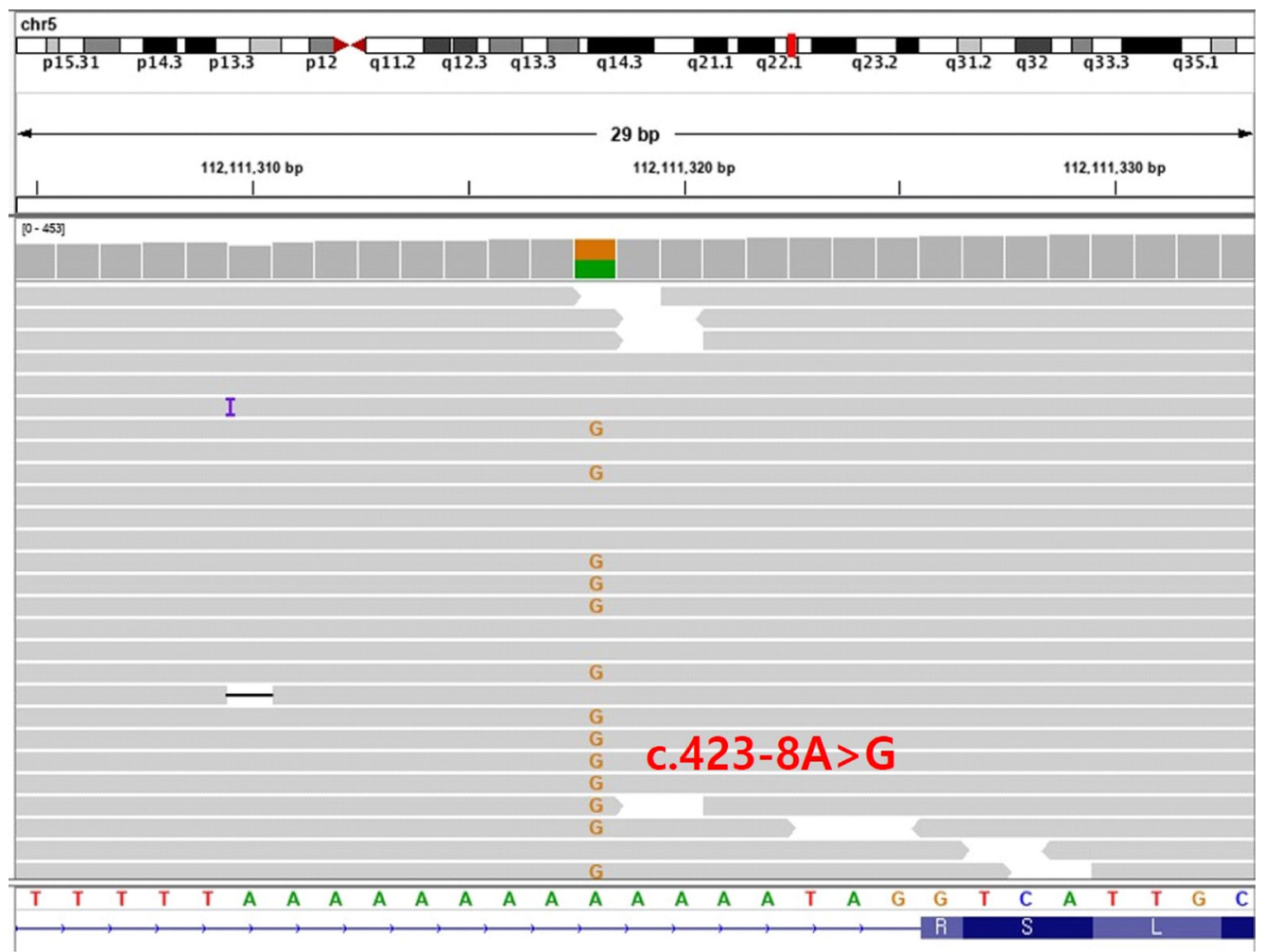

Fig. 1. Next-generation sequencing revealed a variant of $A P C$, involving a substitution of guanine $(G)$ for adenine $(A)$ at the intron located -8 from the acceptor site (c.423-8A $>$ G).

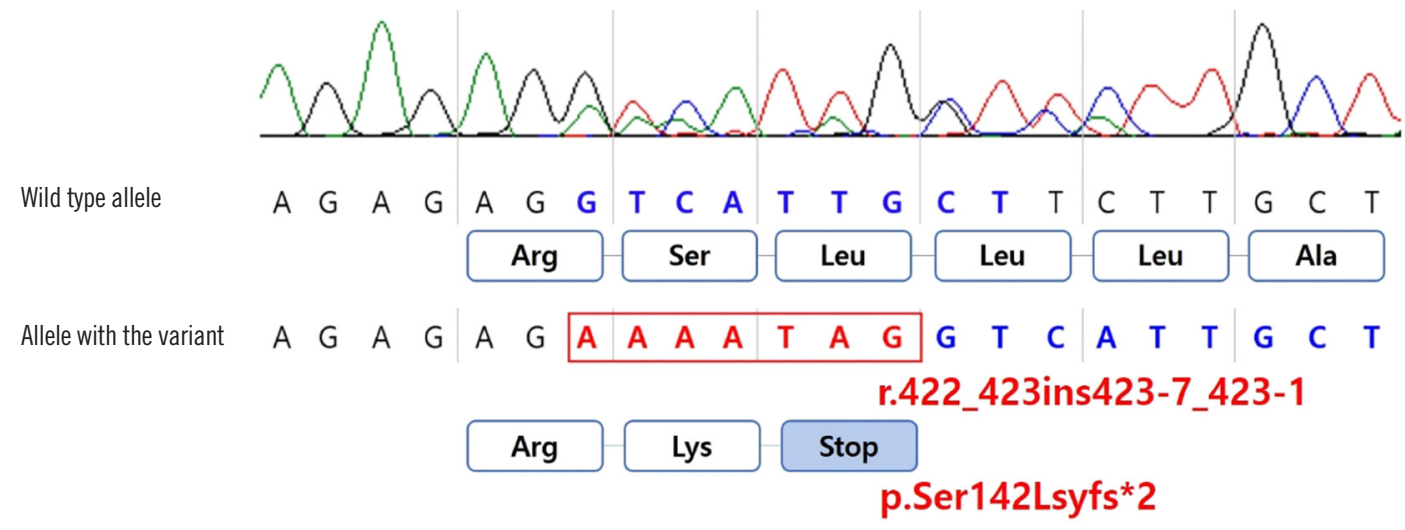

Fig. 2. RNA sequencing revealed an insertion of seven base pairs between r.422 and r.423 (r.422_423ins423-7_423-1). This variant was predicted to cause a premature stop codon (p.Ser142Lysfs*2).

dbscSNV's AdaBoost (adaptive boosting; score: 0.999) [3], Human Splicing Finder [4], and MaxEntScan [5], and Alamut Visual 2 (Interactive Biosoftware, Rouen, France). These algorithms indicated a high chance of splice-site alteration.

As there are numerous adenines close to the acceptor site preceding codon 423 (Fig. 1), presumably, a switch from adenine to guanine at any position in this poly-A site can change the acceptor splice site. The impact of variants on mRNA ex- pression can be highly informative when evaluating their effects at splice junctions and within coding sequences, untranslated regions, and deeper intronic regions [6]. To confirm the splicesite change caused by this variant at the RNA level, we conducted RNA sequencing using primers developed in-house that target the area. RNA was extracted from the patient's leukocytes using High Pure RNA isolation kit (Roche, Indianapolis, IN, USA); RNA was reverse transcribed with RevertAid First Strand cDNA 
Synthesis kit (Thermo Fisher Scientific, Waltham, MA, USA). Sanger sequencing of the cDNA revealed a change of splice acceptor site (r.422_423ins423-7_423-1; Fig. 2). This variant was predicted to cause a premature stop codon (p.Ser142Lysfs ${ }^{*}$ ). Several variants in this poly-A site preceding codon 423 are registered in gene variant databases such as the Human Gene Mutation Database, but they had not been revealed to affect gene or protein function, except the variant reported by Jarry, et al. [7, 8]. These authors reported an APC variant identified as c.423$12 A>G$, which shifted the splice acceptor site up by 11 nucleotides, causing a frameshift and a stop signal 32 codons downstream (p.Ser142Lysfs*32) [8]. So far, studies have not clearly shown an impact of variants between c.423-4 and c.423-16 on gene or protein function $[4,5]$.

Based on the NGS result in our case, only PM2 (absent in population databases) and PP3 (multiple lines of computational evidence support a deleterious effect) evidence could be assigned based on American College of Medical Genetics and Genomics standards and guidelines, classifying it as "uncertain significance" $[3,6]$. However, with the RNA sequencing results added, we could apply PS3 (functional study results) evidence to this variant, classifying it as "likely pathogenic" [6].

Variants in this poly-A site have not been thoroughly validated by RNA sequencing; thus, several variants in this region remain variants of uncertain significance (VUSs), and further evaluation and management of patients with VUSs in this poly-A site and their families may be difficult. With the advancements in sequencing technologies, an increasing number of sequence variants are being detected in the clinical specimens, and the number of VUSs is increasing accordingly. As our study suggests pathogenicity of c.423-8A $>$ G variant via RNA sequencing, we need to make an effort to reduce the number of VUSs by elucidating the impact of sequence variants at the mRNA level when splice-site change is suspected.

\section{ACKNOWLEDGEMENTS}

Nothing to disclose.

\section{AUTHOR CONTRIBUTIONS}

AK wrote the manuscript. YSH, SBL, and JSB treated the patient and collected samples. WL, SC, WKM, HKK, and SA contributed to the interpretation of the results, and the writing and revision of the manuscript.

\section{CONFLICTS OF INTEREST}

The authors declare no conflicts of interest.

\section{RESEARCH FUNDING}

Nothing to disclose.

\section{ORCID}

Aram Kim

Hyun-Ki Kim

Sunyoung Ahn

Yong Sang Hong

Seok-Byung Lim

Jeong-Sik Byeon

Sail Chun

Won-Ki Min

Woochang Lee

https://orcid.org/0000-0003-4210-5313

https://orcid.org/0000-0002-3299-5298

https://orcid.org/0000-0003-3892-0921

https://orcid.org/0000-0001-5672-0072

https://orcid.org/0000-0001-8824-4808

https://orcid.org/0000-0002-9793-6379

https://orcid.org/0000-0002-5792-973X

https://orcid.org/0000-0002-5158-2130

https://orcid.org/0000-0003-3956-6397

\section{REFERENCES}

1. Zhang $Y$, Lu G, Hu Q, Wang X, Li C, Mao Y, et al. A de novo germline mutation of $A P C$ for inheritable colon cancer in a Chinese family using multigene next generation sequencing. Biochem Biophys Res Commun 2014;447:503-7.

2. Stenson PD, Ball EV, Mort M, Phillips AD, Shiel JA, Thomas NS, et al. Human Gene Mutation Database (HGMD): 2003 update. Hum Mutat 2003;21:577-81.

3. Jian $\mathrm{X}$, Boerwinkle $\mathrm{E}$, Liu X. In silico prediction of splice-altering single nucleotide variants in the human genome. Nucleic Acids Res 2014;42: 13534-44.

4. Desmet FO, Hamroun D, Lalande M, Collod-Beroud G, Claustres M, Beroud C. Human Splicing Finder: an online bioinformatics tool to predict splicing signals. Nucleic Acids Res 2009;37:e67.

5. Yeo $\mathrm{G}$ and Burge CB. Maximum entropy modeling of short sequence motifs with applications to RNA splicing signals. J Comput Biol 2004;11: 377-94.

6. Richards S, Aziz N, Bale S, Bick D, Das S, Gastier-Foster J, et al. Standards and guidelines for the interpretation of sequence variants: a joint consensus recommendation of the American College of Medical Genetics and Genomics and the Association for Molecular Pathology. Genet Med 2015;17:405-24.

7. Tsukanov AS, Pospekhova NI, Shubin VP, Kuzminov AM, Kashnikov VN, Frolov SA, et al. Mutations in the APC gene in Russian patients with classic form of familial adenomatous polyposis. Russ J Genet 2017;53: 369-75.

8. Jarry J, Brunet JS, Laframboise R, Drouin R, Latreille J, Richard C, et al. A survey of APC mutations in Quebec. Fam Cancer 2011;10:659-65. 